\title{
Longitudinal drift compression and pulse shaping for high-intensity particle beams
}

\author{
Hong Qin and Ronald C. Davidson \\ Plasma Physics Laboratory, Princeton University, Princeton, New Jersey 08543
}

(Received 20 November 2001; published 7 March 2002)

\begin{abstract}
High beam current can be achieved by longitudinally compressing bunched beams. The objective of drift compression is to compress a long beam bunch by imposing an initial longitudinal velocity distribution over the length of the beam in the beam frame. The longitudinal dynamics of drift compression and pulse shaping for high intensity particle beams are studied using a one-dimensional warm-fluid model. Two self-similar drift compression solutions admitted by the one-dimensional warm-fluid equations are derived. The pulse shaping problem is also solved such that an arbitrary input pulse shape can be shaped into the pulse shapes required by the self-similar drift compression solutions.
\end{abstract}

DOI: 10.1103/PhysRevSTAB.5.034401

PACS numbers: 29.27.Bd, 41.85.Ct, 41.85.Ew

\section{INTRODUCTION}

In accelerators and storage rings, high beam current can be achieved by longitudinally compressing bunched beams. For example, in the currently envisioned configurations for heavy ion fusion, it is necessary to longitudinally compress the beam bunches by a large factor after the acceleration phase and before the beam particles are focused onto the fusion target. In order to obtain enough fusion energy gain, the peak current for each beam is required to be of the order of $10^{3} \mathrm{~A}$ and the bunch length as short as $0.5 \mathrm{~m}$. However, to deliver the beam particles at the required energy, it is both expensive and technically difficult to accelerate short bunches at high current. First, because of the finite rise time of the accelerating waveform, it is much easier to accelerate and transport beam bunches longer than $10 \mathrm{~m}$. Second, short bunches have higher current (density) and therefore stronger space-charge effects which can increase the beam emittance and induce halo particles.

The objective of drift compression [1-7] is to compress a long beam bunch by imposing an initial longitudinal velocity distribution over the length of the beam in the beam frame. As a result, the beam length is reduced as the beam drifts downstream, until the space-charge force in the longitudinal direction becomes strong enough to remove the initial velocity distribution. Different longitudinal compression schemes have been studied in particle simulations [1,2] and in numerical solutions of a cold-fluid model [3-5]. There are also variations in the assumed axial line density profile of the bunched beam. In Haber's study [1], for example, the line density is uniform in the middle of the pulse but falls off at the ends, whereas in the study of Bisognano et al. [3] the line density is parabolic. The quality of drift compression is determined by two factors. A good drift compression scheme should, first of all, work for any reasonable beam pulse shape generated by accelerators, and second, the velocity distribution initially imposed for the purpose of compression should be minimized when the compression is finished. Because the beam length is much shorter after the compression, it is difficult to apply an external field to eliminate the residual longitudinal velocity distribution over the beam length. In desirable drift compression schemes, the spacecharge force and the emittance in the longitudinal direction are utilized to minimize the residual longitudinal velocity distribution.

In this paper, we study the longitudinal drift compression and pulse shaping of high intensity beams using a onedimensional warm-fluid model. We first give two self-similar drift compression solutions. The first solution is characterized by a flattop density profile, a linear velocity profile (i.e., a linear velocity tilt), and a parabolic pressure profile, which will be referred to as the linear selfsimilar drift compression solution. The second solution is characterized by a parabolic density profile, a linear velocity profile, and a double-parabolic pressure profile, which will be referred to as the parabolic self-similar drift compression solution. For the parabolic self-similar drift compression solution, the residual longitudinal velocity distribution can be eliminated by the space-charge force and emittance. We then show how to shape an arbitrary pulse shape into a parabolic form so that it can be subsequently self-similarly compressed after a linear velocity tilt is applied. More generally, we solve the pulse shaping problem. That is, we calculate the initial velocity distribution such that a given initial pulse shape gradually evolves into the desired final pulse shape after a certain length of time.

The paper is organized as follows. In Sec. II, the onedimensional warm-fluid equations with appropriate boundary and initial conditions are introduced as a model to describe the longitudinal dynamics of drift compression and pulse shaping. The self-similar drift compression solutions are derived in Sec. III, and an example from heavy ion fusion is given. The general pulse shaping problem is solved in Sec. IV, illustrated with two examples. 


\section{ONE-DIMENSIONAL WARM-FLUID MODEL FOR LONGITUDINAL DYNAMICS}

We use a one-dimensional warm-fluid model to describe the longitudinal dynamics of drift compression and pulse shaping. For the longitudinal electric field, the conventional $g$-factor model is adopted with axial electric force $\left(e E_{z}\right)$ and geometric factor $(g)$ given by $[5,8]$

$$
e E_{z}=-\frac{g e^{2}}{\gamma^{2}} \frac{\partial \lambda}{\partial z}, \quad g=2 \ln \frac{r_{w}}{r_{b}} .
$$

Here, $e$ is the charge, $\lambda(t, z)$ is the line density, $r_{w}$ is the wall radius, and $r_{b}$ is the average beam radius. We also allow for an externally applied focusing force $F_{z}=-\kappa_{z} z$. In the beam frame moving with axial velocity $\beta c$ relative to the laboratory frame, the warm-fluid equations for the line density $\lambda(t, z)$, average longitudinal velocity $v_{z}(t, z)$, and longitudinal pressure $p_{z}(t, z)$ are given by $[9,10]$

$$
\begin{gathered}
\frac{\partial \lambda}{\partial t}+\frac{\partial}{\partial z}\left(\lambda v_{z}\right)=0 \\
\frac{\partial v_{z}}{\partial t}+v_{z} \frac{\partial v_{z}}{\partial z}+\frac{e^{2} g}{m \gamma^{5}} \frac{\partial \lambda}{\partial z}+\frac{\kappa_{z} z}{m \gamma^{3}}+ \\
\frac{r_{b}^{2}}{m \gamma^{3} \lambda} \frac{\partial p_{z}}{\partial z}=0 \\
\frac{\partial p_{z}}{\partial t}+v_{z} \frac{\partial p_{z}}{\partial z}+3 p_{z} \frac{\partial v_{z}}{\partial z}=0
\end{gathered}
$$

We treat $g$ and $r_{b}$ as constants for present purposes. This assumption simplifies the problem by decoupling the transverse dynamics from the longitudinal dynamics. In reality, when the beam is compressed, the beam radius $r_{b}$ will vary due to the increase of the space-charge force in the transverse direction. However, it is desirable to minimize the variation in $r_{b}$ in order to keep the transverse dimension of the drift compression system at a reasonable size, a goal achievable by applying a nonperiodic focusing lattice with increasing focusing field strength along the beam path. It is possible to design the nonperiodic lattice in such a way that it permits an asymptotically matched beam which has minimal variations in $r_{b}$ [7]. In addition, the effects of the variations in $r_{b}$ are further reduced due to the logarithmic dependence of $g$ and the fact that the space-charge force term in Eq. (3) is smaller than the inertial term and the convection term.

Equations (2)-(4) form a nonlinear hyperbolic system of partial differential equations (PDEs). If $\kappa_{z}$ and $p_{z}$ can be neglected, Eqs. (2) and (3) have the same form as the shallow-water equations. Equation (4) is a simplified version of the pressure equation derived by Lund and Davidson $[9,10]$, and it can be recast into the form

$$
\frac{d}{d t}\left(\frac{p_{z}}{\lambda^{3}}\right) \equiv\left(\frac{\partial v_{z}}{\partial t}+v_{z} \frac{\partial v_{z}}{\partial z}\right)\left(\frac{p_{z}}{\lambda^{3}}\right)=0
$$

In practical laboratory applications, the longitudinal drift compression and pulse shaping are initiated by passing the bunched beam through a voltage gap, which is located at a fixed axial position and has an adjustable voltage shape as a function of time. The typical length of the voltage gap is in the range of centimeters, and the typical voltage applied is hundreds of kilovolts [11]. For most applications, we can safely assume

$$
d \ll z_{b}, \quad v_{z} \ll \beta c,
$$

where $z_{b}$ is the characteristic beam length, and $d$ is the gap length. From Eq. (6), it follows that

$$
\frac{d}{\beta c} \ll \frac{z_{b}}{v_{z}},
$$

which implies that the time it takes for the voltage gap to transfer momentum to the bunched beam is much shorter than the characteristic time of the longitudinal dynamics. We therefore can model the physical effects of a voltage gap by an instantaneously imposed longitudinal velocity distribution across the beam length. By the same argument, a voltage gap can be used to instantaneously remove a longitudinal velocity distribution, and two voltage gaps can be used to replace one velocity distribution by another, as long as Eq. (6) is satisfied. For drift compression applications, these operations can be more easily performed in the upstream region when the beam length is longer and Eq. (6) can be satisfied by a large margin. In terms of the nonlinear PDE system, Eqs. (2)-(4), passing through a voltage gap with a prescribed voltage profile is equivalent to imposing an initial condition on $v_{z}(t, z)$ at $t=0$. Our goal is thus to study, using the one-dimensional warm-fluid model, how the bunched beam is compressed and shaped in the longitudinal direction by imposing different initial velocity distributions.

\section{SELF-SIMILAR DRIFT COMPRESSION SOLUTIONS}

Self-similar drift compression schemes preserve the geometric shape of the bunched beam, as well as the density profile, the pressure profile, and the velocity distribution, while the beam length and amplitudes of the density, velocity, and pressure profiles change with the time. The nonlinear PDE system, Eqs. (2)-(4), admits at least two self-similar drift compression solutions. The first solution is characterized by a flattop density profile and a parabolic pressure profile, and the second solution is characterized by a parabolic density profile and a doubleparabolic pressure profile. Both solutions have a linear velocity distribution (velocity tilt). In the present analysis, we will refer to the first solution as the linear self-similar drift compression solution and to the second solution as the parabolic self-similar drift compression solution.

In the linear self-similar drift compression solution, the $z$ dependence and the $t$ dependence for the field variables are separated according to 


$$
\begin{gathered}
\lambda(t, z)=\lambda_{b}(t), \\
v_{z}(t, z)=-v_{z b}(t) \frac{z}{z_{b}(t)}, \\
p_{z}(t, z)=p_{z b}(t) \frac{z^{2}}{z_{b}^{2}(t)}, \\
\frac{d z_{b}(t)}{d t}=-v_{z b}(t),
\end{gathered}
$$

where $\lambda_{b}(t), z_{b}(t), p_{z b}(t)$, and $v_{z b}(t)$ depend only on $t$. The $z$ dependences of $\lambda(t, z), v_{z}(t, z)$, and $p_{z}(t, z)$ are illustrated in Fig. 1. In the present analysis, we neglect the effects due to the density discontinuity at the beam ends. In general, the discontinuity will propagate into the beam at the plasma wave speed and significantly modify the profile shape. This erosion effect has been experimentally observed [12]. Therefore, the analysis of the linear selfsimilar drift compression solution presented here should only be applied to the central region of the beam in drift compression schemes where the beam has a flat density profile in the middle and a special design shape near the ends to reduce the effects of erosion [1]. A near flattop pulse shape may be favorable in heavy ion fusion applications because it can be focused more easily onto the target. It is interesting to know that such a self-similar flattop solution indeed exists for the fluid equations in the central region. The pulse shape near the ends for this type of drift compression scheme is not expected to be self-similar, but it still can be studied using the one-dimensional fluid equations. This topic is beyond the scope of this paper. In the present analysis, we focus attention on the self-similar flattop region near the center of the beam.

Substituting Eqs. (8) and (9) into the continuity equation (2), we obtain

$$
\frac{d \lambda_{b}}{d t}-\frac{v_{z b}}{z_{b}} \lambda_{b}=0
$$

That is,
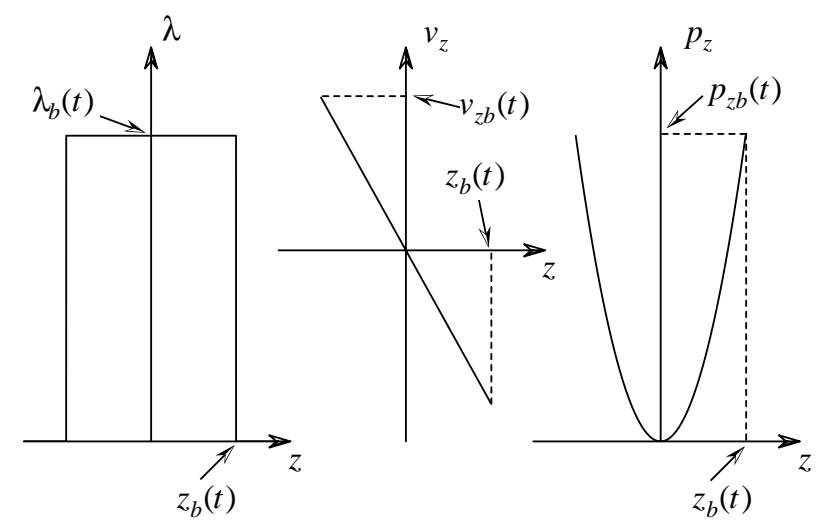

FIG. 1. The $z$ dependences of field variables for the linear selfsimilar drift compression solution.

$$
\frac{1}{\lambda_{b}} \frac{d \lambda_{b}}{d t}+\frac{1}{z_{b}} \frac{d z_{b}}{d t}=0 \Longrightarrow z_{b} \lambda_{b}=\text { const }=N_{b} / 2
$$

where $N_{b}=\int_{-z_{b}}^{z_{b}} d z \lambda(t, z)$ is the total number of particles in the beam pulse. Substituting Eqs. (9) and (10) into the energy equation (4), we obtain

$$
\frac{d}{d t} \ln \left(\frac{p_{z b}}{z_{b}^{2}}\right)+\frac{d}{d t} \ln z_{b}^{5}=0,
$$

and thus

$$
z_{b}^{3} p_{z b}=\text { const }=W .
$$

Similarly, for the momentum equation (3), the $z$ dependence drops out as well, giving

$-\frac{d}{d t}\left(\frac{v_{z b}}{z_{b}}\right)+\frac{v_{z b}^{2}}{z_{b}^{2}}+\frac{\kappa_{z}}{m \gamma^{3}}+\frac{2 r_{b}^{2}}{m \gamma^{3} \lambda_{b}} \frac{p_{z b}}{z_{b}^{2}}=0$.

In terms of $z_{b}$ and $s=\beta c t$,

$$
\frac{d^{2} z_{b}}{d s^{2}}+\frac{\kappa_{z}}{m \gamma^{3} \beta^{2} c^{2}} z_{b}+\frac{\varepsilon_{l}^{2}}{z_{b}^{3}}=0,
$$

where $\varepsilon_{l} \equiv\left(2 r_{b}^{2} W / m \gamma^{3} \beta^{2} c^{2} N_{b}\right)^{1 / 2}$ for the case of the linear self-similar drift compression solution. Equations (13), (15), and (17) describe the dynamics of the time-dependent variables $\lambda_{b}(t), z_{b}(t)$, and $p_{z b}(t)$, and can be solved to give the linear self-similar drift compression solution.

A notable feature of the solution in Eq. (17) is that the beam pressure peaks at the ends of the beam. Therefore, the pressure gradient force is restoring, and the pressure (emittance) term in Eq. (17) compresses the beam. In addition, there is no space-charge force due to the flattop density profile. Both the externally applied force and the pressure-gradient force are focusing. Equation (17) predicts a dramatic compression scenario where the beam longitudinally "implodes" because of the singularity of the (focusing) pressure term in Eq. (17) as $z_{b} \rightarrow 0$. Obviously, this is not a desirable scheme for practical drift compression applications since the amplitude of the longitudinal velocity significantly increases.

We now turn to the parabolic self-similar drift compression solution, which has several desirable features and is a good candidate for practical applications. We consider the class of solution to Eqs. (2)-(4) with

$$
\begin{gathered}
\lambda(t, z)=\lambda_{b}(t)\left(1-\frac{z^{2}}{z_{b}^{2}(t)}\right), \\
v_{z}(t, z)=-v_{z b}(t) \frac{z}{z_{b}(t)}, \\
p_{z}(t, z)=p_{z b}(t)\left(1-\frac{z^{2}}{(t) z_{b}^{2}}\right)^{2}, \\
\frac{d z_{b}(t)}{d t}=-v_{z b}(t) .
\end{gathered}
$$



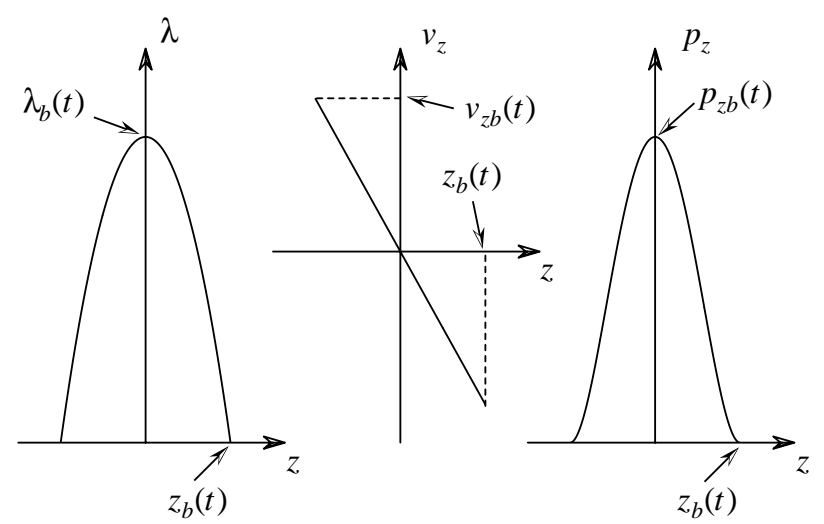

FIG. 2. The $z$ dependences of field variables for the parabolic self-similar drift compression solution.

The $z$ dependences of $\lambda(t, z), v_{z}(t, z)$, and $p_{z}(t, z)$ are illustrated in Fig. 2.

Substituting Eqs. (18)-(20) into Eqs. (2) and (4), we find that the $z$ dependence drops out, and

$$
\begin{gathered}
\frac{d \lambda_{b}}{d t}-\frac{v_{z b}}{z_{b}} \lambda_{b}=0, \\
\frac{d p_{z b}}{d t}-3 \frac{v_{z b}}{z_{b}} p_{z b}=0 .
\end{gathered}
$$

Remarkably, but not surprisingly, for the momentum equation (3), the $z$ dependence also drops out, giving

$$
-\frac{d v_{z b}}{d t}-\frac{e^{2} g}{m \gamma^{5}} \frac{2 \lambda_{b}}{z_{b}}+\frac{\kappa_{z} z_{b}}{m \gamma^{3}}-\frac{4 r_{b}^{2} p_{z b}}{m \gamma^{3} \lambda_{b} z_{b}}=0 .
$$

In general, Eqs. (21)-(24) form a coupled ordinary differential equation system. Most remarkably, these equations recover the longitudinal envelope equation $[8,13]$. From Eqs. (21)-(23), we obtain

$$
\begin{gathered}
\frac{1}{\lambda_{b}} \frac{d \lambda_{b}}{d t}+\frac{1}{z_{b}} \frac{d z_{b}}{d t}=0 \Longrightarrow z_{b} \lambda_{b}=\mathrm{const}=\frac{3}{4} N_{b}, \\
\frac{1}{p_{z b}} \frac{d p_{z b}}{d t}+\frac{3}{z_{b}} \frac{d z_{b}}{d t}=0 \Longrightarrow z_{b}^{3} p_{z b}=\mathrm{const}=W
\end{gathered}
$$

where $N_{b}=\int_{-z_{b}}^{z_{b}} d z \lambda(t, z)$ is the total number of particles in the bunch. Equation (26) implies that $p_{z b}$ increases as $z_{b}$ decreases. The energy equation (4) indicates that the charge bunch is heated in the longitudinal direction when it is compressed. Substituting Eqs. (21), (25), and (26) into Eq. (24) we obtain

$$
\frac{d^{2} z_{b}}{d s^{2}}+\frac{\kappa_{z}}{m \gamma^{3} \beta^{2} c^{2}} z_{b}-\frac{K_{l}}{z_{b}^{2}}-\frac{\varepsilon_{l}^{2}}{z_{b}^{3}}=0,
$$

where $s=\beta c t, K_{l} \equiv 3 N_{b} e^{2} g / 2 m \gamma^{5} \beta^{2} c^{2}$ is the effective longitudinal self-field perveance, and $\varepsilon_{l} \equiv\left(4 r_{b}^{2} W /\right.$ $\left.m \gamma^{3} \beta^{2} c^{2} N_{b}\right)^{1 / 2}$ is the longitudinal emittance for the parabolic self-similar drift compression solution. Equation (27) is the familiar longitudinal envelope equation $[8,13]$. Finally, the solutions for $\lambda_{b}(t), z_{b}(t)$, and $p_{z b}(t)$ can be obtained by solving the longitudinal envelope equation (27) numerically.

The longitudinal envelope equation (27) can be integrated once to give the energy relation

$$
\begin{aligned}
\left(z_{b 0}^{\prime 2}-z_{b}^{\prime 2}\right)= & \frac{\kappa_{z}}{2 m \gamma^{3} \beta^{2} c^{2}}\left(z_{b}^{2}-z_{b 0}^{2}\right) \\
& +2 K_{l}\left(\frac{1}{z_{b}}-\frac{1}{z_{b 0}}\right) \\
& +\varepsilon_{l}^{2}\left(\frac{1}{z_{b}^{2}}-\frac{1}{z_{b 0}^{2}}\right),
\end{aligned}
$$

where $z_{b 0}=z_{b}(s=0)$ and $z_{b 0}^{\prime}=\left[d z_{b} / d s\right]_{s=0}$. Contrary to the linear self-similar drift compression solution, both the space-charge and the emittance terms are defocusing. As a result, the velocity amplitude $v_{z b}=-z_{b}^{\prime}(t)$ decreases as the beam is compressed. It is clear from Eq. (28) that the minimum beam length $z_{b}$ min that can be achieved is given by

$$
\begin{aligned}
z_{b 0}^{\prime 2}= & \frac{\kappa_{z}}{2 m \gamma^{3} \beta^{2} c^{2}}\left(z_{b \min }^{2}-z_{b 0}^{2}\right) \\
& +2 K_{l}\left(\frac{1}{z_{b \min }}-\frac{1}{z_{b 0}}\right) \\
& +\varepsilon_{l}^{2}\left(\frac{1}{z_{b \text { min }}^{2}}-\frac{1}{z_{b 0}^{2}}\right),
\end{aligned}
$$

when $z_{b}^{\prime}=0$.

For given values of the initial and final beam lengths $z_{b f}$ and $z_{b 0}$, we want to minimize the velocity tilt (momentum spread) $\left(z_{b f}^{\prime}, z_{b 0}^{\prime}\right)$ and the beam path length $s_{f}$. However, $z_{b f}^{\prime}, z_{b 0}^{\prime}$, and $s_{f}$ cannot be chosen arbitrarily because of the constraint imposed by the energy relation (28). From Eq. (27), the beam path length required for drift compression can be expressed as

$$
s_{f}=-\int_{z_{b 0}}^{z_{b f}} \frac{d z_{b}}{\sqrt{z_{b 0}^{\prime 2}-\frac{\kappa_{z}}{2 m \gamma^{3} \beta^{2} c^{2}}\left(z_{b}^{2}-z_{b 0}^{2}\right)-2 K_{l}\left(\frac{1}{z_{b}}-\frac{1}{z_{b 0}}\right)-\varepsilon_{l}^{2}\left(\frac{1}{z_{b}^{2}}-\frac{1}{z_{b 0}^{2}}\right)}}
$$

The integral in Eq. (30) can be carried out analytically in closed form when $\kappa_{z}=0$ [14], whereas a numerical evaluation of the integral for the general case is a fairly straightforward task.
As an example of drift compression in a heavy ion fusion driver [15], we consider a $\mathrm{Cs}^{+}$beam with rest mass $m=133 m_{p}$, where $m_{p}$ is the proton mass, kinetic en$\operatorname{ergy}(\gamma-1) m c^{2}=2.5 \mathrm{GeV}$, and initial half-beam length 

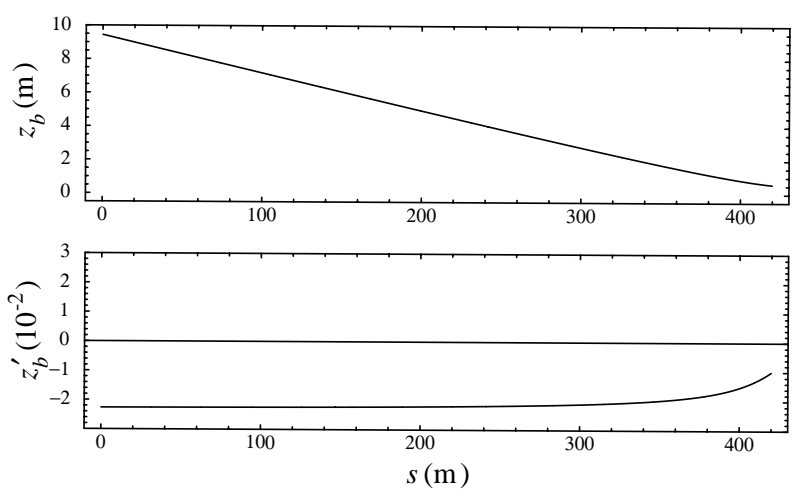

FIG. 3. Longitudinal drift compression of a heavy ion fusion beam.

$z_{b 0}=9.5 \mathrm{~m}$. Our goal is to compress the beam by a factor of 16 , i.e., $z_{b f}=z_{b 0} / 16=0.6 \mathrm{~m}$. The final average current is taken to be $\left\langle I_{f}\right\rangle=2500 \mathrm{~A}$. The longitudinal emittance is taken to be $\varepsilon_{l}=7.7 \times 10^{-6} \mathrm{~m}$, and effective longitudinal self-field perveance $K_{l}=1.3 \times 10^{-4} \mathrm{~m}$ with $g=2.0$. Assuming no external focusing $\left(\kappa_{z}=0\right)$ and $z_{b 0}^{1}=-0.023$, we obtain $s_{f}=421.5 \mathrm{~m}$ by evaluating the integral in Eq. (30), and $z_{b f}^{\prime}=-0.01$ by solving Eq. (28). The beam half-length $z_{b}(s)$, obtained numerically from the longitudinal envelope equation (27), is plotted together with the velocity tilt $z_{b}^{\prime}(s)$ in Fig. 3 .

In this example, the velocity amplitude is almost a constant until near the end of the drift compression, where the beam has been compressed by a large factor and the space-charge force becomes significant.

\section{LONGITUDINAL PULSE SHAPING}

The parabolic self-similar drift compression solution described in Sec. III requires the initial beam pulse shape to be parabolic. However, the beam pulse shape is generally not parabolic after the acceleration phase in practical accelerator applications. It is necessary to shape the beam pulse into a parabolic form before imposing a velocity tilt. In the subsequent analysis, longitudinal pressure effects will be omitted because in many applications, such as the case studied in Sec. III for heavy ion fusion, the pressure effects are negligible. The external focusing force will also be omitted in the subsequent analysis. In general, the pulse shaping problem can be posed as finding the initial velocity distribution $V(z) \equiv v_{z}(t=0, z)$ such that a given initial pulse shape $\Lambda(z) \equiv \lambda(t=0, z)$ evolves into a given final pulse shape $\Lambda_{T}(z) \equiv \lambda(t=T, z)$ at time $t=T$. In particular, we need to solve the pulse shaping problem for the case where $\Lambda_{T}(z)$ is a parabolic function of $z$ for the scheme of parabolic self-similar drift compression.

When studying the pulse shaping problem, it is convenient to choose the following normalized variables:

$$
\bar{v}_{z}=\frac{v_{z}}{\beta c}, \quad \bar{z}=\frac{z}{z_{b 0}}, \quad \bar{\lambda}=\frac{\lambda}{\lambda_{b 0}}, \quad \bar{t}=\frac{t \beta c}{z_{b 0}},
$$

where $z_{b 0}$ is the initial beam half-length and $\lambda_{b 0}$ is the initial beam line density at the beam center $(z=0)$. In the normalized variables, the one-dimensional fluid equations, neglecting pressure effects and external focusing, are given by

$$
\begin{gathered}
\frac{\partial \lambda}{\partial t}+\frac{\partial}{\partial z}\left(\lambda v_{z}\right)=0, \\
\frac{\partial v_{z}}{\partial t}+v_{z} \frac{\partial v_{z}}{\partial z}+\bar{K}_{l} \frac{\partial \lambda}{\partial z}=0
\end{gathered}
$$

where $\bar{K}_{l} \equiv \lambda_{b 0} e^{2} g / m \gamma^{5} \beta^{2} c^{2}$ is the normalized longitudinal perveance. In Eqs. (32) and (33), we dropped the overbar notation for the normalized variables for simplicity.

As noted in Sec. III, the space-charge force is important only near the end of drift compression. Since pulse shaping is expected to be performed in the upstream region where the pulse length is long enough for condition (6) to be satisfied, $\bar{K}_{l}$ will be treated as a small parameter in the following study. We first solve the pulse shaping problem to zeroth order in $\bar{K}_{l}$, and then carry out the analysis to determine the first-order corrections. To order lowest order, neglecting the $\bar{K}_{l}$ term in Eq. (33), the momentum equation is decoupled from the continuity equation, which gives

$$
\begin{aligned}
& \frac{\partial \lambda}{\partial t}+\frac{\partial}{\partial z}\left(\lambda v_{z}\right)=0 \\
& \frac{\partial v_{z}}{\partial t}+v_{z} \frac{\partial v_{z}}{\partial z}=0
\end{aligned}
$$

Equations (34) and (35) can be solved by integrating along characteristics. On the characteristics defined by

$$
C: \frac{d z}{d t}=v_{z}
$$

Eqs. (34) and (35) are

$$
\begin{gathered}
\frac{d \lambda}{d t}=-\lambda \frac{\partial v_{z}}{\partial z} \\
\frac{d v_{z}}{d t}=0
\end{gathered}
$$

Because $d v_{z} / d t=0$ on $C$, the family of characteristics $C$ are straight lines in the $(t, z)$ plan, which can be represented as

$$
C: z=\xi+V(\xi) t
$$

where

$$
V(\xi) \equiv v_{z}(t=0, \xi)
$$

The solution for $v_{z}(t, z)$ can be formally written as

$$
v_{z}(t, z)=V(\xi(t, z))
$$

where $\xi(t, z)$ as a function of $t$ and $z$ is determined from Eq. (39). 
From Eqs. (41) and (39), four useful identities can be derived, i.e.,

$$
\begin{aligned}
\frac{\partial \xi}{\partial z} & =\frac{1}{1+V^{\prime}(\xi) t} \\
\frac{\partial \xi}{\partial t} & =\frac{-V(\xi)}{1+V^{\prime}(\xi) t} \\
\frac{\partial v_{z}}{\partial z} & =\frac{V^{\prime}(\xi)}{1+V^{\prime}(\xi) t} \\
\frac{\partial v_{z}}{\partial t} & =\frac{-V(\xi) V^{\prime}(\xi)}{1+V^{\prime}(\xi) t}
\end{aligned}
$$

From Eqs. (37) and (44) we obtain

$$
\frac{d \ln \lambda}{d t}=\frac{-V^{\prime}(\xi)}{1+V^{\prime}(\xi) t} \text { on } C .
$$

Since $\xi$ is a constant on $C$, Eq. (46) can be integrated to give

$$
\begin{aligned}
\ln \lambda & =\ln \lambda(t=0, \xi)+\int_{0}^{t} \frac{-V^{\prime}(\xi)}{1+V^{\prime}(\xi) t} d t \\
& =\ln \Lambda(\xi)+\ln \left[1+V^{\prime}(\xi) t\right]
\end{aligned}
$$

where $\Lambda(z) \equiv \lambda(t=0, z)$ is the initial line density profile. The solution to Eq. (46) for $\lambda(t, z)$ is

$$
\lambda(t, z)=\frac{\Lambda(\xi)}{1+V^{\prime}(\xi) t} .
$$

For the pulse shaping problem, the final line density profile $\Lambda_{T}(z) \equiv \lambda(t=T, z)$ is specified. We therefore obtain

$$
\Lambda_{T}(z)=\Lambda_{T}[\xi+V(\xi) T]=\frac{\Lambda(\xi)}{1+V^{\prime}(\xi) T},
$$

which can be viewed as an ordinary differential equation for $V(\xi)$. It can be simplified using the variable $U(\xi)$ defined by

$$
U(\xi) \equiv \xi+V(\xi) T .
$$

In terms of $U(\xi)$, Eq. (49) becomes

$$
\Lambda_{T}(U) d U=\Lambda(\xi) d \xi .
$$

Finally, $U(\xi)$ is determined by solving Eq. (51) for the given functional forms $\Lambda_{T}(z)$ and $\Lambda(z)$, and with the appropriate boundary conditions. We find that $V(\xi)$ is simply related to $U(\xi)$ by

$$
V(\xi)=\frac{U(\xi)-\xi}{T} .
$$

Note that $U(\xi)$ does not depend on $T$, and $V(\xi)$ is linearly proportional to $1 / T$. To shorten the beam path length for pulse shaping, we need only to linearly increase the amplitude of the initial velocity distribution $V(\xi)$.

We consider two examples with the following symmetries:

$$
\begin{gathered}
v_{z}(t,-z)=-v_{z}(t, z), \\
\lambda(t,-z)=\lambda(t, z),
\end{gathered}
$$

which imply the boundary conditions for $V(\xi)$ and $U(\xi)$ corresponding to

$$
\begin{aligned}
& V(\xi=0)=0, \\
& U(\xi=0)=0 .
\end{aligned}
$$

Example 1-Pulse shaping without compression: We first consider the case where

$$
\begin{array}{r}
\Lambda(z)= \begin{cases}1-z^{m}, & 0 \leq z \leq 1, \\
0, & 1<z, \\
\Lambda(-z), & z<0,\end{cases} \\
\Lambda_{T}(z)= \begin{cases}\left(1-z^{n}\right) \frac{m(n+1)}{n(m+1)}, & 0 \leq z \leq 1, \\
0 & 1<z, \\
\Lambda(-z), & z<0 .\end{cases}
\end{array}
$$

Here, $m, n \neq-1$, and the coefficient $m(n+1) /$ $n(m+1)$ in the expression for $\Lambda_{T}(z)$ assures the conservation of the total number of particles. Equation (51) can integrated to give

$$
\left[U(\xi)-\frac{U(\xi)^{n+1}}{n+1}\right] \frac{m(n+1)}{n(m+1)}=\xi-\frac{\xi^{m+1}}{m+1} .
$$

The parabolic self-similar drift compression solution corresponds to $n=2$. In this case, there are three solutions for $U(\xi)$. The solution satisfying the boundary condition (56) is

$$
U(\xi)=-\frac{1-i \sqrt{3}+\sqrt[3]{-2} p^{2}}{\sqrt[3]{4} p}
$$

where

$$
\begin{gathered}
p=\sqrt[3]{-3 a+\sqrt{-4+9 a^{2}}}, \\
a=\frac{2(m+1)}{3 m}\left(\xi-\frac{\xi^{m+1}}{m+1}\right) .
\end{gathered}
$$

For a large value of $m \gg 1, \Lambda(z)$ has a flattop shape with a fast falloff near the ends of the pulse. Equations (60) and (52) then give the initial velocity distribution $V(z)$ necessary to shape a flattop bunched beam into a parabolic shape which can be self-similarly compressed after imposing a linear velocity tilt. In Fig. $4, \Lambda_{T}(z)=(45 / 32)\left(1-z^{2}\right)$ and $\Lambda(z)=1-z^{15}$ are plotted versus $z$, together with $V(z)$. 

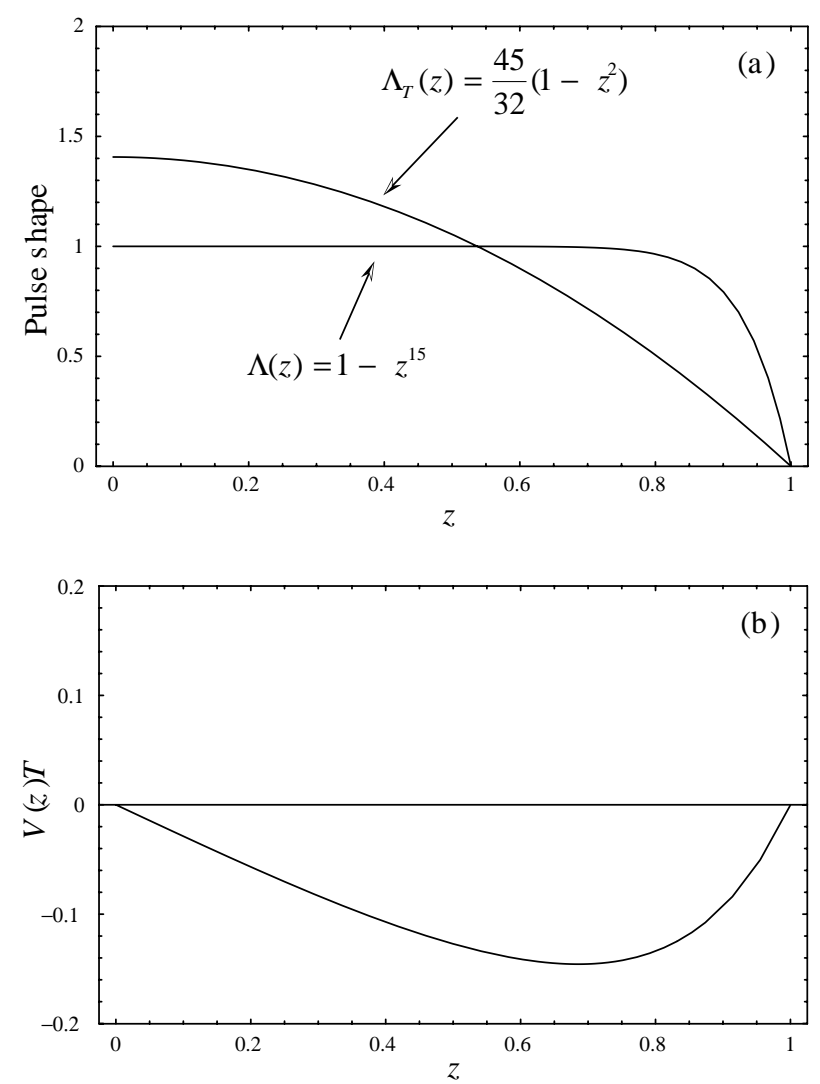

FIG. 4. Initial pulse shape $\Lambda(z)=1-z^{15}$ and final pulse shape $\Lambda_{T}(z)=(45 / 32)\left(1-z^{2}\right)$ are plotted in (a). The initial velocity $V(z)$ given by Eqs. (56) and (52) is plotted in (b).

Example 2-Pulse shaping with compression: In this example, a beam pulse is compressed while being shaped. We consider the case where

$$
\begin{gathered}
\Lambda(z)= \begin{cases}1-z^{m}, & 0 \leq z \leq 1, \\
0, & 1<z, \\
\Lambda(-z), & z<0,\end{cases} \\
\Lambda_{T}(z)= \begin{cases}{\left[1-(\alpha z)^{n}\right] \frac{\alpha m(n+1)}{n(m+1)},} & 0 \leq z \leq \frac{1}{\alpha}, \\
0, & \frac{1}{\alpha}<z, \\
\Lambda(-z), & z<0,\end{cases}
\end{gathered}
$$

where $\alpha>1$ is the compression factor. Again, the coefficient $\propto m(n+1) / n(m+1)$ in the expression for $\Lambda_{T}(z)$ assures the conservation of the total number of particles. Equation (51) can be integrated to give

$$
\left\{\alpha U(\xi)-\frac{[\alpha U(\xi)]^{n+1}}{n+1}\right\} \frac{m(n+1)}{n(m+1)}=\xi-\frac{\xi^{m+1}}{m+1}
$$

which is identical to Eq. (59) if $\alpha U(\xi)$ is replaced by $U(\xi)$. It is easy to verify that $\alpha U(\xi=1)=1$ and therefore

$$
V(\xi=1)=\frac{(1 / \alpha-1)}{T}
$$

For the case of a beam being shaped but not compressed, $\alpha=1$ and $V(\xi=1)=0$. When $\alpha>1$, the beam is simultaneously being shaped and compressed, and $V(\xi=$ $1)<0$. An inward velocity at the beam ends is needed to compress the beam. Plotted in Fig. 5 are $\Lambda(z), \Lambda_{T}(z)$, and $V(z)$ for the case where $m=15, n=2$, and $\alpha=3$.

To complete the study on pulse shaping, we now carry out the analysis to $O\left(\bar{K}_{l}\right)$. We express

$$
\begin{gathered}
\lambda(t, z)=\lambda_{0}(t, z)+\lambda_{1}(t, z), \\
v_{z}(t, z)=v_{z 0}(t, z)+v_{z 1}(t, z) .
\end{gathered}
$$

Here, $\lambda_{0}(t, z)$ and $v_{z 0}(t, z)$ are the leading-order solutions obtained in Eqs. (48) and (41). To $O\left(\bar{K}_{l}\right)$, Eqs. (32) and (33) can be expressed as

$$
\begin{aligned}
\left(\frac{d}{d t}\right)_{0} \lambda_{1} & =\frac{\partial \lambda_{1}}{\partial t}+v_{z 0} \frac{\partial \lambda_{1}}{\partial z} \\
& =-\lambda_{1} \frac{\partial v_{z 0}}{\partial z}-\frac{\partial}{\partial z}\left(\lambda_{0} v_{z 1}\right), \\
\left(\frac{d}{d t}\right)_{0} v_{z 1} & =\frac{\partial v_{z 1}}{\partial t}+v_{z 0} \frac{\partial v_{z 1}}{\partial z} \\
& =-v_{z 1} \frac{\partial v_{z 0}}{\partial z}-\bar{K}_{l} \frac{\partial \lambda_{0}}{\partial z}
\end{aligned}
$$
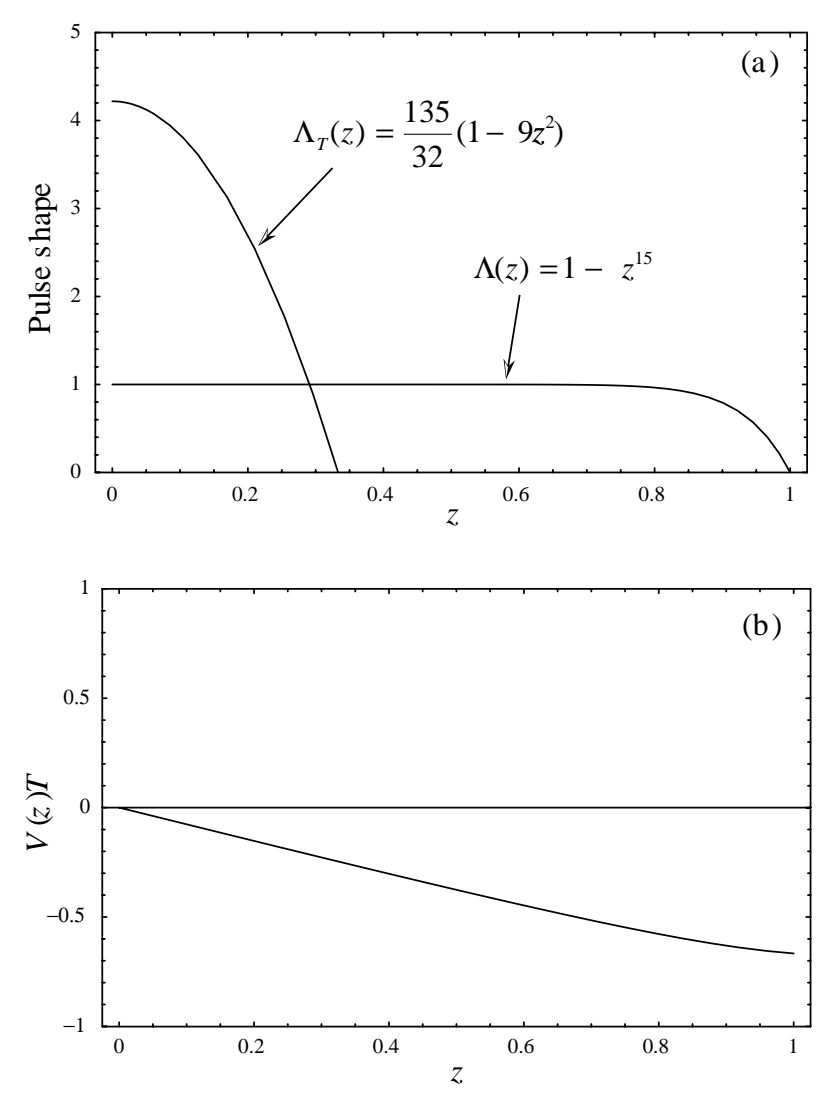

FIG. 5. Initial pulse shape $\Lambda(z)=1-z^{15}$ and final pulse shape $\Lambda_{T}(z)=(135 / 32)\left(1-9 z^{2}\right)$ are plotted in (a). The initial velocity $V(z)$ given by Eqs. (56) and (52) is plotted in (b). 
In the above equations, $\left(\frac{d}{d t}\right)_{0}=\frac{\partial}{\partial t}+v_{z 0} \frac{\partial}{\partial z}$ is the total time derivative along the unperturbed characteristics, i.e.,

$$
C_{0}: z=\xi+V_{0}(\xi) t
$$

Equation (70) is an inhomogeneous ordinary differential equation for $v_{z 1}$ on the unperturbed characteristics $C_{0}$. The solution to the corresponding homogeneous equation is

$$
v_{z 1}(t, z)=\frac{V_{1}(\xi)}{1+V_{0}^{\prime}(\xi) t}
$$

where $V_{1}(\xi) \equiv v_{z 1}(t=0, \xi)$, and $\xi(t, z)$ is determined as a function of $t$ and $z$ from Eq. (71). Using the method of variational coefficients, the solution to Eq. (70) is found to be

$$
v_{z 1}=\frac{1}{1+V_{0}^{\prime}(\xi) t}\left\{V_{1}(\xi)-\bar{K}_{l} \frac{\partial}{\partial \xi}\left(\frac{\Lambda_{0}(\xi)}{V_{0}^{\prime}(\xi)} \ln \left[1+V_{0}^{\prime}(\xi) t\right]\right)\right\} .
$$

In deriving Eq. (73), use has been made of

$$
\frac{\partial \lambda_{0}}{\partial z}=\frac{\partial \lambda_{0}}{\partial \xi} \frac{\partial \xi}{\partial z}=\frac{\partial \lambda_{0}}{\partial \xi} \frac{1}{1+V_{0}^{\prime}(\xi) t} .
$$

By the same procedure, Eq. (69) can be integrated to give

$$
\begin{aligned}
\lambda_{1}= & \frac{\Lambda_{1}(\xi)}{1+V_{0}^{\prime}(\xi) t}-\frac{1}{1+V_{0}^{\prime}(\xi) t} \frac{\partial}{\partial \xi} \\
& \times\left\{\frac{\Lambda_{0}(\xi) V_{1}(\xi) t}{1+V_{0}^{\prime}(\xi) t}-\bar{K}_{l} \Lambda_{0}(\xi) \frac{\partial}{\partial \xi}\left[\frac{\Lambda_{0}(\xi)}{V_{0}^{\prime}(\xi)}\right] \frac{V_{0}^{\prime}(\xi) t-\ln \left[1+V_{0}^{\prime}(\xi) t\right]}{\left[1+V_{0}^{\prime}(\xi) t\right]^{2}}-\bar{K}_{l} \frac{\Lambda_{0}^{2}(\xi)}{V_{0}^{\prime}(\xi)} V_{0}^{\prime \prime}(\xi) \frac{t^{2}}{\left[1+V_{0}^{\prime}(\xi) t\right]^{2}}\right\}
\end{aligned}
$$

At time $t=T$, we obtain

$$
\Lambda_{T}(z)=\lambda_{0}(t=T, z)+\lambda_{1}(t=T, z) .
$$

Since $\Lambda_{T}(z)$ and $\Lambda(z)$ are prescribed in the pulse shaping problem, we take $\Lambda_{T 1}(z)=0$ and $\Lambda_{1}(z)=0$. This results in

$$
V_{1}(\xi)=\bar{K}_{l} \frac{\partial}{\partial \xi}\left[\frac{\Lambda_{0}(\xi)}{V_{0}^{\prime}(\xi)}\right] \frac{V_{0}^{\prime}(\xi)-\ln \left[1+V_{0}^{\prime}(\xi) T\right] / T}{1+V_{0}^{\prime}(\xi) T}+\bar{K}_{l} \frac{\Lambda_{0}(\xi)}{V_{0}^{\prime}(\xi)} V_{0}^{\prime \prime}(\xi) \frac{T}{1+V_{0}^{\prime}(\xi) T}+c^{\prime} .
$$

The constant $c^{\prime}$ is determined from the boundary condition for $V_{1}(\xi)$. For cases with the symmetries in Eqs. (53) and (54), $c^{\prime}=0$ because $V_{0}^{\prime}(\xi)$ and $\Lambda_{0}(\xi) / V_{0}^{\prime}(\xi)$ are even functions and their first derivatives vanish at $\xi=0$.

From Eq. (77), the amplitude of the first-order correction $V_{1}(\xi)$ increases with $T$. To be precise, $V_{1}(\xi)$ is linearly proportional to $\bar{K}_{l} T$ for small $V_{0}^{\prime}(\xi) T$. This dependence is the result of the accumulation of spacecharge-induced corrections over time. To reduce the spacecharge-induced corrections, we can increase the amplitude of $V_{0}(\xi)$ so that $T$, the duration of the pulse shaping, is reduced.

\section{CONCLUSIONS AND FUTURE WORK}

To summarize, we studied the longitudinal dynamics of drift compression and pulse shaping using a onedimensional warm-fluid model. It was found that at least two self-similar drift compression solutions exist for the one-dimensional warm-fluid equations: the linear selfsimilar drift compression solution and the parabolic selfsimilar drift compression solution. Detailed analysis showed that the latter solution has several desirable features and is a good candidate for practical drift compression schemes. We also solved the pulse shaping problem perturbatively in the weak space-charge limit, such that an arbitrary pulse shape produced after the acceleration phase can be shaped into the pulse shapes required by the self-similar drift compression solutions. Besides the two self-similar drift compression solutions derived here, the one-dimensional warm-fluid equations may admit other self-similar drift compression solutions that can be used in drift compression applications. A systematic method to discover families of self-similar drift compression solutions will provide more possibilities for different drift compression applications. In addition, extending the solution of the pulse shaping problem to the strong space-charge region will enable us to modify the pulse shape over the entire beam path with more accuracy. In this region, it is necessary to solve the fluid equations numerically to study the influence of space charge on the profile evolution. An efficient algorithm using Lagrangian coordinates is being developed. New results in these areas will be reported in future publications.

\section{ACKNOWLEDGMENTS}

This research was supported by the U.S. Department of Energy under the auspices of the Virtual National Laboratory for Heavy Ion Fusion. It is a pleasure to acknowledge the benefit of useful discussions with John Barnard, Irving Haber, Ed Lee, and Simon Yu. 
[1] I. Haber, in Proceedings of the Symposium on Accelerator Aspects of Heavy Ion Fusion, Darmstadt, 1982 (GSI, Darmstadt, 1982), p. 372.

[2] I. Hofmann and I. Bozsik, in Proceedings of the Symposium on Accelerator Aspects of Heavy Ion Fusion, Darmstadt, 1982 (Ref. [1]), p. 362.

[3] J. Bisognano, E. P. Lee, and J. W.-K. Mark, LLNL Report No. 3-28, 1985.

[4] D. D.-M. Ho, S. T. Brandon, and E. P. Lee, Part. Accel. 35, 15 (1991).

[5] M.J.L. de Hoon, Ph.D. thesis, University of California, Berkeley, 2001.

[6] E. P. Lee and J. J. Barnard, in Proceedings of the 2001 Particle Accelerator Conference, Chicago (to be published), p. 2928.

[7] H. Qin, C. Jun, R. C. Davidson, and P. Heitzenroeder, in Proceedings of the 2001 Particle Accelerator Conference,
Chicago (to be published), p. 1761.

[8] D. Neuffer, Part. Accel. 11, 23 (1980).

[9] S. M. Lund and R. C. Davidson, Phys. Plasmas 5, 3028 (1998).

[10] R.C. Davidson and H. Qin, Physics of Intense Charged Particle Beams in High Energy Accelerators (World Scientific, Singapore, 2001), pp. 409-414.

[11] S. Yu (private communication).

[12] D.X. Wang, in Proceedings of the 1995 Particle Accelerator Conference, Dallas, Texas (IEEE, Piscataway, NJ, 1995), p. 48.

[13] F. J. Sacherer, IEEE Trans. Nucl. Sci. 18, 1105 (1971); see also Physics of Intense Charged Particle Beams in High Energy Accelerators (Ref. [10]), pp. 128-140.

[14] J. J. Barnard (private communication).

[15] E. P. Lee (private communication). 